\title{
External Quality Assurance Scheme in a National Reference Laboratory for HIV Testing in South India
}

\author{
Kamala Mary Sushi, Thatchinamoorthy Gopal, Saramma Mini Jacob, Ganesan Arumugam, \\ Anitha Durairaj
}

Department of Experimental Medicine, TN Dr. MGR Medical University, Chennai, India.

Email: *saramini@yahoo.com

Received May $17^{\text {th }}, 2012$; revised July $10^{\text {th }}, 2012$; accepted July $18^{\text {th }}, 2012$

\begin{abstract}
Background: The Department of Experimental Medicine functions as the National Reference Laboratory (NRL) for HIV testing covering 11 Medical Colleges (State Reference Laboratories SRL) \& 723 subcenters i.e. Integrated Counseling \& Testing Centres and Blood Banks. The External Quality Assurance Scheme (EQAS) in NRL implements Quality Control (QC) Testing, Proficiency panel testing and training programs. Materials \& Method: 9419 samples (4393 HIV negative/5026 HIV positive) were tested for QC. All the samples were tested using HIV rapid test (CombAids) and HIV positives alone were tested using Tridot and EIA Comb. The QC samples consisted of $20 \%$ negative and all positives. All the 723 subcenters were provided with 5 coded plasma samples ( 3 reactive \& 2 negative) for proficiency testing using rapid tests. The aliquot panel $(500 \mu \mathrm{l})$ were provided twice a year for testing to monitor the laboratory performance. Results: Out of 9419 samples tested for QC, $9371(99.49 \%)$ reported correct results and $48(0.50 \%)$ discordant results. Out of 48 samples $26(0.27 \%)$ were false positives and $22(0.23 \%)$ false negative. Mislabeling, sample contamination, leaking vials, transcriptional errors, tests that were not performed correctly were identified. For proficiency testing $91.8 \%$ reported test results. $645(97.13 \%)$ reported correct results \& $19(2.86 \%)$ incorrect results. Out of 19 samples $7(1.05 \%)$ were false positive \& $12(1.80 \%)$ were false negative. Hands on training were provided and the 19 discordant centers reported correct results on retesting. Conclusion: Significant progress in establishing a well coordinated HIV Laboratory network of NRL and SRLs had been developed. However the HIV testing and Quality Assurance needs to be strengthened towards certification.
\end{abstract}

Keywords: Quality Assurance; HIV Testing; South India

\section{Introduction}

Quality Assurance is the series of procedures that ensure that a correct result is achieved in a standard, reproducible and traceable manner [1]. It is achieved by a series of processes that assure the most accurate and highest quality result. To achieve a high quality result input from all members of every laboratory in a testing network is required. All laboratory personnel should be aware of the necessity for quality performance. This requires continuous education throughout the testing system.

The Department of Experimental Medicine of the TN Dr. MGR Medical University functions as one of the National Reference Laboratory (NRL) in the External Quality Control Scheme (EQAS) under the National AIDS Control Organization (NACO), Government of India for HIV testing. The NRL is in charge of eleven State Reference Laboratories (SRL) and 723 subcenters

${ }^{*}$ Corresponding author. i.e., Intergrated Counselling and Testing Centers (ICTC) and Blood Banks (BB).

The NRL tests Quality Control (QC) samples i.e., every 20th HIV negative and all positives. Apart from QC testing, preparation of panels, hands on training and training programs on EQAS and QC testing for sentinel surveillance are the other activities.

The Diagnostic tests to detect antibodies to HIV have sensitivity and specificity which are not absolute. In all these tests we have false negative and false positive results which are inherent and cannot be avoided. Thus the validity of diagnostic test results is dependent to a very large extent on the quality of the technical conditions under which the tests are performed. Meaning thereby, consistent reproduction of reliable results requires a stringent overall Quality Assurance program which would control technical conditions before, during and after each assay.

The Center for Disease control (CDC) developed guidelines for implementing and operating QA programs. 
It recommends testing sites participate in external quality assessments ie Proficiency testing of panels, competency assessments and QA monitoring by outside organization observing the testing [2]. The National Reference Laboratory (NRL) implements the External Quality Assurance Program for HIV testing to provide standard quality results.

\section{Material \& Methods}

This is a retrospective study. The Quality Control (QC) samples were received from the 11 State Reference Laboratories (SRL) by courier service and hand delivery from 2007 to 2008. The SRLs test the samples in their respective centers. After testing the QC samples alone are sent to the NRL. Every $20^{\text {th }}$ HIV negative sample and all HIV positive samples from the State Reference Laboratories are tested as QC samples in the NRL. 9419 samples were received in the NRL for QC testing (5026 positive and 4393 negative). In QC testing all the samples are retested using CombAids (Span Diagnostics Ltd, Surat, India) as the $1^{\text {st }}$ rapid kit. It employs the same principle as EIA thereby the immobilized antigen antibody complex is visualized by means of colour producing (chromogenic reaction). Each comb was observed for the control and the test results. The HIV positives alone were retested by the $2^{\text {nd }}$ kit HIV Tridot (J. Mitra \& Co, India) and the $3^{\text {rd }}$ rapid EIA comb (J. Mitra \& Co). NACO strategy of using 3 rapids for HIV testing was followed [3]. Insufficient and hemolysed samples, samples that were not properly labeled were excluded and informed to the testing centers. Apart from the routine samples and kit controls Internal Quality Control samples (known HIV positive \& negative) were used during each run. The test kits were provided by the APSACS (Andhra Pradesh State AIDS Control Society). All discordant samples were discussed with the testing center and were followed up with hands on training for the laboratory technicians. It is a continuous process where the NRL plays a key role in monitoring and evaluating the SRLs.

Apart from QC testing two training programs were conducted twice a year for the SRLs. One Microbiologist and one laboratory technician represented each SRL. The training comprises lectures, hands on training in HIV testing and panel distribution. The SRL's \& 723 subcentres were provided with 5 panels ( $3 \mathrm{HIV}$ positives \& 2 negative coded samples) during the training program. Panels were prepared by NRL procuring blood bags or plasma from blood banks. The samples were characterized using ELISA and HIV rapid tests. The panels were validated by sending them to the Apex Laboratory before the training program. A report was sent from the Apex Lab to NRL informing the results and samples that had concordant results were used for proficiency panel testing. Aliquots were further prepared and stored in $-80^{\circ} \mathrm{C}$.
The SRL's in turn conducted a one day training program for their sub centers and provided the panels. The reports of the 723 subcenters provided a feedback and this enabled the NRL to train them periodically and improve their performance in HIV testing.

\section{Results}

Nine thousand four hundred and nineteen Quality control (QC) samples were tested at the National Reference Laboratory during a period of 2 years. Out of 9419, 5026 samples were HIV positives (53.3\%) and 4393 were HIV negative samples. The samples were retested using three different HIV rapids test kits. The 9419 samples were tested using Comb AIDS kit as the first rapid kit.

Out of 9419 samples tested, 9371 reported concordant results. 4367 QC samples were HIV negative and 5004 QC were positive for HIV. 48 reported discordant results. 26 samples were false positive and 22 were false negatives (Table 1). Out of 5026 QC positive samples reported from the SRL, 22 samples were false negative and 5004 were HIV positive. Similarly out of 4393 QC negative samples 26 tested false positive and the remaining 4367 were negative (Table 2).

The QC positive samples, 26 false positive and 22 false negative samples were retested using the second HIV rapid test Tridot. 5004 samples were positive. 26 reported false positive and 22 false negative in Tridot. The above QC samples were again retested with the third HIV rapid test kit and similar results were observed. 48 discordant samples reported the same results in all the three rapid kits. The QC sample result \& discordant samples were reported to the respective centers. The main reasons for discordant samples were contamination of samples, pipette tip contamination, labeling error, leaking vials and transcriptional errors.

Table 1. Concordant and discordant samples tested for Quality Control.

\begin{tabular}{|c|c|c|c|}
\hline Total no of QC & Concordant & \multicolumn{2}{|c|}{ Discordant samples } \\
\hline \multirow[t]{3}{*}{9419} & $9371(99.4 \%)$ & \multicolumn{2}{|c|}{$48(0.50 \%)$} \\
\hline & & False positive & False negative \\
\hline & & 26 & 22 \\
\hline
\end{tabular}

Table 2. Samples tested at SRL and NRL.

\begin{tabular}{|c|c|c|c|c|c|c|c|}
\hline \multicolumn{4}{|c|}{ SRL } & \multicolumn{4}{|c|}{ NRL } \\
\hline \multicolumn{2}{|c|}{ No. of samples } & \multicolumn{2}{|c|}{ CombAids } & \multicolumn{2}{|c|}{ TRIDOT } & \multicolumn{2}{|c|}{ EIAComb } \\
\hline \multirow[t]{2}{*}{ HIV+ } & HIV- & $\mathrm{HIV}+$ & HIV- & $\mathrm{HIV}+$ & HIV- & $\mathrm{HIV}+$ & HIV- \\
\hline & 4393 & 26 & 4367 & 26 & & 26 & \\
\hline 5026 & & 5004 & 22 & 5004 & 22 & 5004 & 22 \\
\hline
\end{tabular}


Twice a year proficiency testing panels (coded samples) were distributed to SRL's during the training program. Out of 723 subcenters, $664(91.8 \%)$ reported the panel results to the NRL. $59(8.16 \%)$ centers did not send the report. Out of 664 subcentres, 645 (97.1\%) reported correct results and $19(2.86 \%)$ reported incorrect results. Out of 19 samples $7(1.05 \%)$ reported false positive \& 12 $(1.80 \%)$ false negative. Contamination of samples was the major reason for incorrect results. Hands on training on HIV testing were provided to the laboratory technicians in 19 centers as follow up. New set of plasma panels ( 5 Nos) were provided. All the 19 centres reported correct results.

\section{Discussion}

The NRL is involved in Quality Control testing, proficiency panels, conducting training programs and providing hands on training for HIV testing. It is also involved in sentinel surveillance QC testing which is conducted annually by NACO. Quality Assurance program ensures high level of performance of HIV assays [4]. As a NRL the Quality Assurance program ensures the participating centers are correctly testing, getting consistent results and it checks their validity of results.

In India the EQAS program had been implemented by NACO since 2000. It functions with 1 Apex Laboratory and 13 National Reference Laboratories and thousands of sub centers covering the entire Government Medical Colleges, Hospitals, blood banks and primary health centers in each state. This EQAS program is linked with the respective State AIDS Control Society (SACS) in each state for the effective functioning under the NACO.

Out of 9419 samples tested for QC, 48 samples had discordant results: 26 false positive and 22 false negative. In the follow up training program, they were instructed to follow the Standard Operating Procedures (SOP), use of correct pipetting technique, use of single tip for each sample, avoid transcriptional errors and prevent sample contamination. Participation in proficiency testing is a key component of any Laboratory Quality assurance program, whether available locally, nationally or internationally [5].

Quality Assurance program should be in place to continuously assess and improve the performance of Laboratory results. It is important for the physicians in guiding the patient for treatment and for further management. All aspects of sample handling right from the arrival to reporting must be monitored, documented and subjected to quality control procedures. It is also suggested that regular audits of laboratory procedures and reviewing incident reports must be carried out by the senior staff who are in charge and should be discussed with the Director or the Head of the Laboratory $[5,6]$.

The Standard Operating Procedures (SOP) is an im- portant component of the Quality Assurance program. All the members of the laboratory should be familiar with the procedures. The SOP's are reviewed every year and no deviations from the procedures in order to get correct results.

Reagent and equipment performance must be monitored over time to detect any changes in quality and integrity [7]. The storage of reagents and expiry date too plays an important role in the performance of testing. If controls are not working faint spots or lines may develop. It will lead to misinterpretation of results. Calibrating pipettes and centrifuges annually should be done in the laboratory. The temperatures of the refrigerators are monitored by a digital thermometer where HIV testing kits are stored. Expired kits should not be used.

In Catalonia, Spain a survey on HIV testing was undertaken to assess the quality of HIV testing. HIV specimens were identified by specific labels, extracting new specimens for a second test to confirm, or failing to guarantee the confidentiality of results was found [8]. In a QA program the pretesting, testing and post testing should be monitored, observed and evaluated. The recommendations will play an important role in improving the centers.

In China the National AIDS Reference Laboratory (NARL) provides quality assurance through technical, bio safety and managerial trainings, periodic proficiency testing, on site supervisory inspections and commercial serological kit evaluations [9]. The 9419 samples tested for QC provided a feedback mechanism on the performance of the respective centers. It provided an opportunity to pick up discordant samples or it could have been left undetected. After the implementation of the EQAS program the standards of HIV testing had been improved.

Rapid test with immediate test result are popular in the US and Canada. It requires good laboratory quality control practices. The laboratory must be backed by a license to provide confirmation of positive results and resolution for indeterminate results. Quality Assurance and participation in HIV proficiency testing ensures the accurate, timely and clinically relevant laboratory results [7]

The EQAS training twice a year and proficiency testing was aimed to monitor testing at SRL's who in turn apply the same methods to the subcenters. Out of 723 subcenters provided with proficiency panel, 664 (91.8\%) reported the results. $8.16 \%$ did not report. The main reason being the center did not participate in the training program. $97.1 \%$ reported correct results. 19 (2.86\%) reported incorrect results. Hands on training were provided to 19 centers and they were provided with new set of proficiency panels. All the 19 centers reported correct results.

In Poland there is no guidelines related to HIV diagnosis quality assurance and control. Hence developing of 
a National Unified quality control system based with a central institution is highly desirable. This was based on the survey conducted to assess the laboratory performance. It was also suggested certification be made mandatory for all diagnostic laboratories [10]. The aim was to improve the reliability of results among the clinicians and the patients.

The proficiency testing provided an opportunity for the subcenters to know their abilities and improve their performance. They were also provided with forms to fill up the results ie, kit name, lot No, expiry dates, results of controls and samples and the final interpretation of results. These forms were provided by the Apex Laboratory and it provided an opportunity for the Lab personnel to read and fill in the forms. All the 723 subcenters were provided with unique codes identifying with the SRL, District and their VCTC, BB centers.

In India five laboratories were identified as Regional Institutes (RIs) to monitor and supervise the Quality control practices and assurances in the sentinel surveillance testing covering five states. The testing laboratories were adhering to the standard Operating Procedures. Concordance of test result between the RI and laboratories was high. The lacunae and the recommendations were put forward for the future surveillance [11].

\section{Conclusions}

Significant progress had been made in establishing a well coordinated HIV Laboratory network through EQAS. In this program it ensures mistakes may be avoided by proper sample handling, labeling, testing and reporting. The consistency of performance is maintained. The Quality Assurance system with proficiency testing, quality control testing, training programs and on-site monitoring strengthens the participating laboratories and provides opportunity for improvement.

NABL certification is in process in the EQAS program. The certification process which covers the entire laboratory performance will provide opportunities for filling the gaps and strengthening their performance.

\section{Acknowledgements}

The authors are thankful to the National AIDS Control Organization and Andhra Pradesh State AIDS Control Society for supplying HIV tests kits.

\section{REFERENCES}

[1] E. M. Dax, "External Quality Assurance Scheme in HIV testing Guidelines," National Reference Laboratory, Melbourne, 2000. www.nrl.gov.au

[2] Centers for Disease Control and Prevention, "Quality Assurance Guidelines for Testing Using Rapid HIV Antibody Tests Waived under the Clinical Laboratory Improvement Amendments of 1988," 2007. http:www.cdc.gov/hiv/topics/testing/resources/guidelines/ pdf/QA_Guidlines.pdf

[3] National AIDS Control Organisation, "Guidelines for HIV Testing," 2007. www.nacoonline.org

[4] D. E. Dwyer, "Advances in HIV Laboratory Testing," NSW Public Health Bulletin, Vol. 21, No. 3-4, 2010, pp. 86-88. doi:10.1071/NB10018

[5] D. W. Tholen, L. M. Berte, D. J. Boone, W. G. Cooper, J. Gun-Munro, M. A. Noble, S. J. Sarewitz and M. L. Williams, "Using Proficiency Testing to Improve the Clinical Laboratory, Approved Guideline," CLSI/NCCLS GP27-A, Vol. 27, No. 8, 1999.

[6] G. B. Clarke, S. J. Sarewitz, K. J. Aziz, K CastanedaMendez, L. K. Miller. E. Simson and D. W. Tholen, "Continuous Quality Improvement: Essential Management Approaches: Approved Guideline," CLSI/NCCLS GP22-A, Vol. 19, No. 13, 1999.

[7] M. Fearon, "The Laboratory Diagnosis of HIV Infections," The Canadian Journal of Infectious Diseases and Medical Microbiology, Vol. 16, No. 1, 2005, pp. 26-30.

[8] M. J. Casado, A. Rovira, C. Blanch and J. Casabona, "Survey of the Performance of Anti-HIV Antibody Detection Tests in Laboratories in Catalonia (Spain)," Gac Sanit Journal, Vol. 18, No. 1, 2004, pp. 68-71. doi: $10.1157 / 13057982$

[9] Y. Jiang, M. Qiu, G. Zhang, W. Xing, Y. Xiao, P. Pan, J. Yao, C. Y. Ou and X. Su, "Quality Assurance in the HIV/AIDS Laboratory Network of China," International Journal of Epidemiology, Vol. 39, Suppl. 2, 2010, pp. 7278. doi:10.1093/ije/dyq224

[10] M. Parczewski, K. Madalinski, M. Leszozyszyn-Pynka and A. Boron-Kaczmarska, "Evaluation of Quality of HIV Diagnostic Procedures in Poland," Przegi Epidemiol Journal, Vol. 64, No. 1, 2010, pp. 47-53.

[11] S. Kant, K. Goswami, S. K. Rai, L. Dar and P. Misra, "Quality Assurance of Annual HIV Sentinel Surveillance: Experience of Regional Institute, Central Zone," Indian Journal of Public Health, Vol. 51, No. 1, 2007, pp. 28-32. 